\title{
A sustained-release formulation of doxycycline hyclate (Atridox) prevents simultaneous infection of Anaplasma phagocytophilum and Borrelia burgdorferi transmitted by tick bite
}

Correspondence

Nordin S. Zeidner

Naz2@cdc.gov

Received 25 July 2007

Accepted 21 December 2007

\author{
Nordin S. Zeidner, ${ }^{1}$ Robert F. Massung, ${ }^{2}$ Marc C. Dolan, ${ }^{1}$ Eric Dadey, ${ }^{3}$ \\ Elizabeth Gabitzsch, ${ }^{1}$ Gabrielle Dietrich ${ }^{1}$ and Michael L. Levin ${ }^{2}$ \\ ${ }^{1}$ Division of Vector-Borne Infectious Diseases, Centers for Disease Control and Prevention, \\ Fort Collins, CO 80522, USA \\ ${ }^{2}$ Division of Viral and Rickettsial Diseases, Centers for Disease Control and Prevention, Atlanta, \\ GA 30333, USA \\ ${ }^{3}$ OLT Laboratories, Fort Collins, CO 80525, USA
}

Current prophylaxis for infected tick bites consists of personal protective measures directed towards ticks. This study compared the efficacy of a single oral dose of doxycycline with that of a single injection of sustained-release doxycycline in a model of Lyme borreliosis and Anaplasma phagocytophilum infection. Dosages of doxycycline were equilibrated based on previously determined peak plasma levels in mice [oral, $2.4 \mu \mathrm{g}(\mathrm{ml} \text { plasma })^{-1}$; sustained release, $1.9 \mu \mathrm{g}(\mathrm{ml}$ plasma $)^{-1}$ ] determined $8 \mathrm{~h}$ after inoculation. In challenge experiments where five Borrelia burgdorferi-infected and five $A$. phagocytophilum-infected nymphs were used per mouse, only 20 and $30 \%$ of mice were protected from $B$. burgdorferi and $A$. phagocytophilum infection, respectively, using oral doxycycline. In contrast, $100 \%$ of mice receiving sustained-release doxycycline were protected from $A$. phagocytophilum infection, as indicated by real-time PCR of blood samples, quantitative PCR and culture isolation of spleen samples, and protected against B. burgdorferi infection as demonstrated by culture of ear, heart and bladder. Although 15-40 copies of $A$. phagocytophilum could be amplified from the spleens of mice treated with sustained-release doxycycline, no viable $A$. phagocytophilum from these spleens could be cultured in HL-60 cells. In contrast, 7/10 mice receiving oral doxycycline were PCR- and culture-positive for $A$. phagocytophilum, with copy numbers ranging from 800 to 10000 within the spleen, as determined by quantitative PCR. Other correlates with A. phagocytophilum infection included a significant difference in spleen mass (mean of $110 \mathrm{mg}$ for sustained-release treatment versus a mean of $230 \mathrm{mg}$ for oral treatment) and the number of splenic lymphoid nodules (mean of 8 for sustained-release treatment versus mean of 12.5 for oral doxycycline) as determined by histopathology. These studies indicate that a single injection of a sustained-release formulation antibiotic may offer a viable prophylactic treatment option for multiple infectious agents in patients presenting with tick bites.

\section{INTRODUCTION}

Lyme borreliosis is the most common vector-borne disease reported in the USA (CDC, 2004). Human granulocytic anaplasmosis (formerly known as human granulocytic ehrlichiosis) is an emerging tick-borne disease first described in the midwestern USA, and now routinely found along the north-eastern seaboard and upper midwestern states (Bakken \& Dumler, 2000; McQuiston et al., 1999). The aetiological agent of human granulocytic anaplasmosis is

Abbreviation: CDC, Centers for Disease Control and Prevention.
Anaplasma phagocytophilum and is transmitted in these parts of the USA by Ixodes scapularis, the same tick vector that transmits Lyme disease in these geographical regions (Massung et al., 2004; Pancholi et al., 1995). Both of these zoonotic pathogens are maintained in cycles comprising I. scapularis ticks and Peromyscus leucopus reservoir mice (Stafford et al., 1999). Infection with one of these agents may accompany infection of the other, both in vector ticks and reservoir hosts (Adelson et al., 2004; Stafford et al., 1999). Moreover, within endemic areas, humans may acquire combined infections, leading to overlapping disease symptomatology (Aguero-Rosenfeld et al., 2002; Krause et al., 
2002). Clinical delineation of this becomes important, as acknowledging dual infection may affect the inclusion of appropriate and effective chemotherapy for A. phagocytophilum, which may be different from treatment for acute Lyme borreliosis (Maurin et al., 2003; Nadelman et al., 1997).

Doxycycline is the drug of choice for treating patients with acute A. phagocytophilum infection. Likewise, although amoxicillin is widely used to treat acute Lyme borreliosis, studies indicate that doxycycline may be as effective (Wormser et al., 2003). Similarly, some success has been obtained using a single dose $(200 \mathrm{mg})$ of doxycycline hyclate for prophylactic treatment of people exposed to an $I$. scapularis tick bite (Nadelman et al., 2001). Our laboratory has reported that delivery of a single dose of a sustainedrelease formulation of doxycycline hyclate is $100 \%$ effective in preventing tick-transmitted Borrelia burgdorferi infection in a murine model of Lyme borreliosis (Zeidner et al., 2004a). Moreover, similar results have been reported in a murine model of tick-transmitted A. phagocytophilum (Massung et al., 2005). Compared with the rapid clearance of orally delivered doxycycline, sustained-release doxycycline plasma levels in these studies were sustained over a 19 day period after delivery at a concentration below the MIC reported for B. burgdorferi (Johnson et al., 1990) and with no apparent toxicity. In general, controlled-release delivery systems for antimicrobial agents have been shown to increase the bioavailability of short-lived antibiotics to mammalian tissues and to enhance treatment efficacy (Matschke et al., 2002).

In contrast to previous studies looking at treatment of a single infectious agent transmitted by ticks, the current studies were done to compare the effectiveness of a single oral administration of doxycycline hyclate with a single subcutaneous administration of sustained-release doxycycline to prevent dual transmission of $A$. phagocytophilum and $B$. burgdorferi simultaneously delivered by I. scapularis ticks.

\section{METHODS}

Tick transmission of B. burgdorferi and A. phagocytophilum. Laboratory-reared, B. burgdorferi-infected I. scapularis nymphal ticks were raised as described by Piesman (1993) and have been shown previously to be free of A. phagocytophilum and Babesia microti (Zeidner et al., 2004a). These ticks were infected with low-passagenumber B. burgdorferi strain B31. Likewise, laboratory-reared nymphal $I$. scapularis ticks that had previously fed as larvae on $P$. leucopus mice infected with a low-passage-number of A. phagocytophilum Webster strain (Massung et al., 2004) were used for tick inoculation studies. In dual-infection studies, a total of ten infected nymphs (five of each infected with B. burgdorferi or A. phagocytophilum) were placed on the head and neck areas of specific-pathogenfree, 6 -week-old female $\mathrm{C} 3 \mathrm{H} / \mathrm{HeJ}$ mice ( $n=5$ per group, mass equal to $20.1 \pm 0.2$ g; Jackson Laboratory). In single infection control studies, only five infected nymphs harbouring either B. burgdorferi or A. phagocytophilum were placed on mice. These studies were then repeated with $n=5$ mice per group. At $72 \mathrm{~h}$ after tick infestation, the partially engorged ticks were removed from all mice as described previously (Massung et al., 2005; Zeidner et al., 2004a). The mice were then randomly assigned to receive either $2 \mathrm{mg}$ oral doxycycline hyclate in water, $4.2 \mathrm{mg}$ sustained-release doxycycline hyclate copolymer formulation (Atridox) (QLT Laboratories), or water or DLlactide in $\mathrm{N}$-methyl-2-pyrrolidone co-polymer (QLT Laboratories) vehicle treatment controls. These dosages of doxycycline were previously shown to deliver equivalent peak levels of drug in mouse plasma measured $8 \mathrm{~h}$ after inoculation (Zeidner et al., 2004a). Oral doxycycline was delivered by gavage in $0.1 \mathrm{ml}$ tissue-grade water. The sustained-release doxycycline hyclate (Atridox) was mixed according to the manufacturer's specifications and transferred to a $1 \mathrm{ml}$ Luer Lock syringe (Becton Dickinson) fitted with a 25 -gauge needle for subcutaneous injection. A total of $0.05 \mathrm{ml}$ was then delivered to each mouse along the dorsal midline of the animal between the scapulae.

Determination of B. burgdorferi and A. phagocytophilum infection in mice. Mice were bled weekly for 3 weeks post-tick infestation to determine the A. phagocytophilum infection status in blood. These EDTA-treated blood samples were frozen at $-80{ }^{\circ} \mathrm{C}$ until analysed. At 3 weeks post-treatment, all mice were euthanized and skin (ear biopsy), heart and bladder were cultured in Barbour-StennerKelly medium (Schwartz et al., 1992) to determine the B. burgdorferi infection status (Sinsky \& Piesman, 1989). Samples were also placed in tissue fixative (Streck Laboratories) to determine histopathology. Likewise, spleen samples were taken to determine A. phagocytophilum infection levels, both by PCR and by direct culture using HL-60 cells. All spleens were first weighed, and equal amounts of tissue were either frozen at $-80{ }^{\circ} \mathrm{C}$ until analysed by PCR, or homogenized and cocultured on human promyelocytic HL-60 cells. The remainder of the spleen was then placed into tissue fixative for histopathological evaluation. Growth of A. phagocytophilum in HL-60 cells was monitored by light microscopy after methanol fixation of cytocentrifuge preparations and staining with the Diff-Quik diagnostic stain (Dade Behring).

DNA extraction and quantification of $\boldsymbol{A}$. phagocytophilum copy number. A real-time PCR assay (Massung et al., 2004, 2005) was utilized to determine the presence of A. phagocytophilum in peripheral blood, and a quantitative PCR assay was used to determine $A$. phagocytophilum copy number in spleen samples. DNA was extracted from tissues as described previously (Massung et al., 2004, 2005) using a DNeasy tissue kit (Qiagen). In the case of blood, $200 \mu \mathrm{l}$ whole blood per mouse was used, and copy numbers in spleen were determined [copy number ( $\mathrm{mg}$ spleen tissue $)^{-1}$ ]. The real-time PCR assay utilized primers and probe to amplify the spacer region between the single-copy $23 \mathrm{~S}$ and $5 \mathrm{~S}$ rRNA genes as described previously (Massung et al., 2004). Real-time PCR assays were performed on each sample in triplicate and the mean was determined per sample. Both positive and negative controls were included in all PCR assays.

Determination of histopathology. Tissue sections were prepared as described previously (Zeidner et al., 2004b). Briefly, specimens placed in Streck's tissue fixative were subjected to standard processing, embedded in paraffin and sectioned at $5 \mu \mathrm{m}$. Sections were then stained with haematoxylin and eosin for standard light microscopy evaluation. All tissue sections were read and analysed in a coded, blind fashion.

Statistical analysis. Fisher's test was utilized to determine significant differences among treatment groups. Student's $t$-test was used to determine significant differences in splenic copy numbers of A. phagocytophilum among treatment groups. In both cases, $P<0.05$ was considered a statistically significant difference between groups.

\section{RESULTS}

As shown in Table 1, sustained-release doxycycline hyclate completely protected mice (100\%) from both B. burgdorferi 
Table 1. Treatment efficacy of sustained-release doxycycline hyclate against $B$. burgdorferi co-transmitted with $A$. phagocytophilum

\begin{tabular}{|lcccc|}
\hline & $\begin{array}{c}\text { No. of mice infected with } \\
\text { Bb/no. tested }\end{array}$ & $\begin{array}{c}\text { Protection } \\
(\%)^{*}\end{array}$ & $\begin{array}{c}\text { No. of mice infected with } \\
\text { Ap/no. tested }\end{array}$ & $\begin{array}{c}\text { Protection } \\
(\%) \dagger\end{array}$ \\
\hline Sustained-release doxycycline & $0 / 10$ & 100 & $0 / 10$ & 100 \\
DL-Lactide co-polymer control & $7 / 10$ & $\mathrm{NA}$ & $7 / 10$ & $\mathrm{NA}$ \\
Oral doxycycline & $8 / 10$ & 20 & $7 / 10$ & 30 \\
Water control & $10 / 10$ & $\mathrm{NA}$ & $8 / 10$ & $\mathrm{NA}$ \\
\hline
\end{tabular}

Ap, A. phagocytophilum; Bb, B. burgdorferi; NA, not applicable.

${ }^{\star}$ Culture results of skin biopsy analysed at 28 days, and heart and bladder at 56 days post-tick infestation.

$\dagger$ Results of quantitative PCR analysis and culture (spleen) at 21 days post-tick infestation.

and A. phagocytophilum infection transmitted simultaneously by infected ticks. In comparison, 70 and $80 \%$ of mice receiving a single dose of oral doxycycline became infected with either A. phagocytophilum or B. burgdorferi, respectively, which was not statistically different from either the co-polymer or water control mice $(P=0.2)$. In the case of B. burgdorferi, infection status was based on culture of skin (ear), heart and bladder. Where animals were infected with B. burgdorferi, all three tissues were consistently infected and demonstrated pathology of the heart, spleen and bladder (data not shown). In the case of $A$. phagocytophilum infection, A. phagocytophilum DNA could not be detected within the peripheral blood on days 7,14 and 21 in mice receiving sustained-release doxycycline, and that were infected with A. phagocytophilum alone or simultaneously challenged with B. burgdorferi (Fig. 1a). In contrast, DNA could be amplified from the blood of 7/10 mice receiving oral doxycycline on days 7, 14 and 21 (Fig. 1a). As previous studies have indicated that the spleen is a site of $A$. phagocytophilum replication and accumulation, we analysed the spleen on day 21 by quantitative, real-time PCR and by direct isolation of A. phagocytophilum from cultured HL-60 cells. As shown in Fig. 1(b), mice challenged with $A$. phagocytophilum-infected ticks alone or simultaneously with B. burgdorferi-infected ticks, and treated with Atridox, demonstrated mean values of 21.6 and 15.3 copies (mg spleen $)^{-1}$, respectively, as measured by quantitative PCR. These copy numbers were significantly different from those in mice treated with oral doxycycline (dual infection, mean of 987 copies $\mathrm{mg}^{-1}, P<0.001$; A. phagocytophilum infection alone, mean of 3892 copies $\left.\mathrm{mg}^{-1}, P<0.001\right)$. Moreover, there was no statistically significant difference in A. phagocytophilum splenic copy number between mice treated with oral doxycycline and the co-polymer or water control (dual infection, mean of 987 versus 1181 copies $\mathrm{mg}^{-1}, P=0.76 ; A$. phagocytophilum infection alone, mean of 3892 versus 768 copies $\left.\mathrm{mg}^{-1}, P=0.24\right)$. Attempts to culture A. phagocytophilum from spleen homogenates from mice treated with sustained-release doxycycline hyclate showed no growth, suggesting that the PCR-positive spleens from these mice did not contain viable organisms (data not shown).

Previous studies have indicated that replication and accumulation of $A$. phagocytophilum occurs within the spleen (Massung et al., 2004, 2005). As shown in Fig. 2, a marked difference in the size of spleens was noted when comparing mice treated with oral doxycycline with those

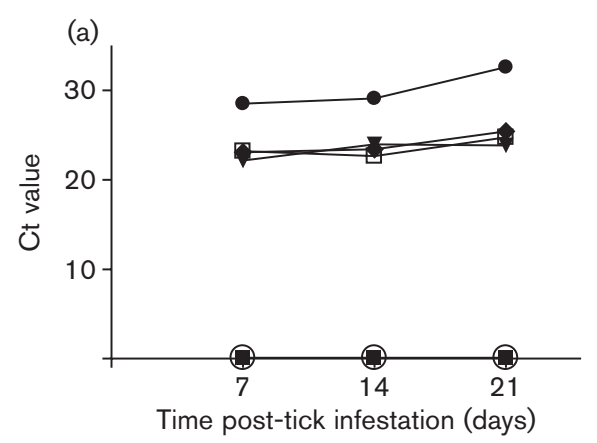

(b)

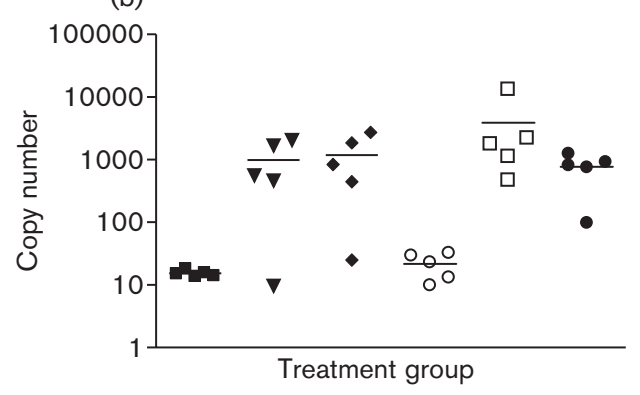

- Ap+Bb (Atridox)

$\checkmark \mathrm{Ap}+\mathrm{Bb}$ (oral doxycycline)

- Ap+Bb (co-polymer C)

- Ap (Atridox)

$\square$ Ap (oral doxycycline)

- Ap (water C)

Fig. 1. (a) PCR amplification of $A$. phagocytophilum from the blood of tick-infested mice. Blood samples were harvested on days 7,14 and 21 post-tick infestation for quantitative real-time PCR analysis as described in Methods. Points represent the mean values per treatment group $(n=10)$ of mice. (b) Representative copy numbers of $A$. phagocytophilum PCR-amplified from the spleen of mice 21 days after tick infestation. Lines represent the mean copy number value per treatment group. Ap, A. phagocytophilum; $\mathrm{Bb}$, B. burgdorferi. 
(a)

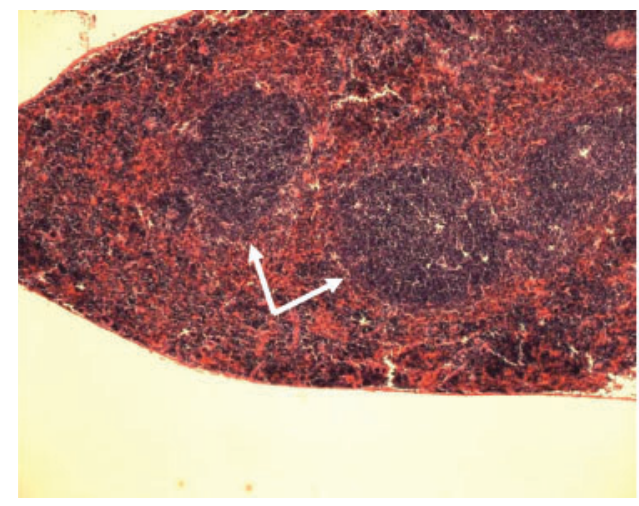

Sustained-release doxycycline (b)

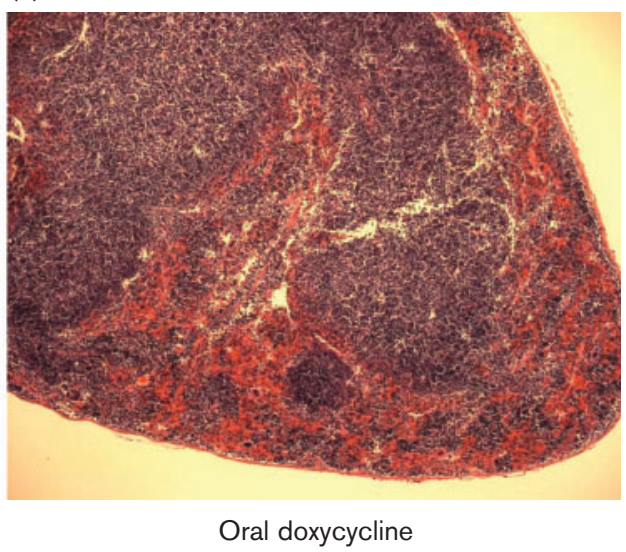

Fig. 2. Histopathology following dual B. burgdorferi and A. phagocytophilum challenge. (a) A representative spleen sample (magnification $\times 5$ ) of an animal treated with sustained-release doxycycline hyclate (Atridox). Arrows indicate discrete lymphoid follicles. (b) A representative tissue sample (magnification $\times 5$ ) of an animal treated with oral doxycycline, which was both PCRand culture-positive for $A$. phagocytophilum, as well as culture-positive for $B$. burgdorferi.

treated with Atridox. Marked lymphoid hyperplasia and an increase in cellular infiltrate (monocytes, neutrophils and plasma cells) within the red pulp were noted for animals treated with oral doxycycline who were both PCR- and culture-positive for A. phagocytophilum (Fig. 2b). In contrast, those animals in which $A$. phagocytophilum and $B$. burgdorferi infection was prevented retained a normal splenic architecture with discrete lymphoid follicles and no extraneous cellular infiltrate within the red pulp (Fig. 2a). Moreover, gross spleen mass was significantly different when comparing treatment groups $(n=10)$. Those animals in which infection was prevented by treatment with Atridox demonstrated spleen masses ranging from 98.6 (dual infection) to $121 \mathrm{mg}$ (A. phagocytophilum infection alone) similar to normal $(n=10) \mathrm{C} 3 \mathrm{H} / \mathrm{HeJ}$ spleens (mean of $107 \pm 0.2 \mathrm{mg}, P=0.28$ ) (Fig. 3a). In contrast, animals treated with oral doxycycline had spleen masses ranging from 213 to $244 \mathrm{mg}$ (Fig. 3a), which was statistically significant $(P<0.0005)$. Moreover, there was no difference in spleen masses of animals treated with oral doxycycline compared with the water- or co-polymer-treated controls $(P=0.51)$. We noted that the increase in spleen mass could be due to the marked lymphoid hyperplasia in PCR-positive spleens, as well as the number of lymphoid nodules enumerated (Fig. 3b). In Atridox-treated mice, which resisted infection, the mean number of lymphoid nodules was 7.6, equivalent to the number of nodules found in normal $\mathrm{C} 3 \mathrm{H} / \mathrm{HeJ}$ mice (mean of $7.0 \pm 0.3, P=0.51$ ) (Fig. 3b). In contrast, in animals treated with oral doxycycline or vehicle treatment controls, which did not resist infection, the average number of lymphoid nodules ranged from 11.9 to $12.6(P<0.001)$. As with spleen mass, there was no statistically significant difference between animals treated with oral doxycycline and those receiving vehicle controls (Fig. 3b), whether water or co-polymer $(P=0.13)$.

\section{DISCUSSION}

Previous studies in our laboratory have demonstrated that a single injection of a sustained-release form of doxycycline hyclate completely protects mice from tick-transmitted B. burgdorferi infection (Zeidner et al., 2004a). A second study in our laboratory indicated $100 \%$ protection against tick-transmitted $A$. phagocytophilum infection in a similar

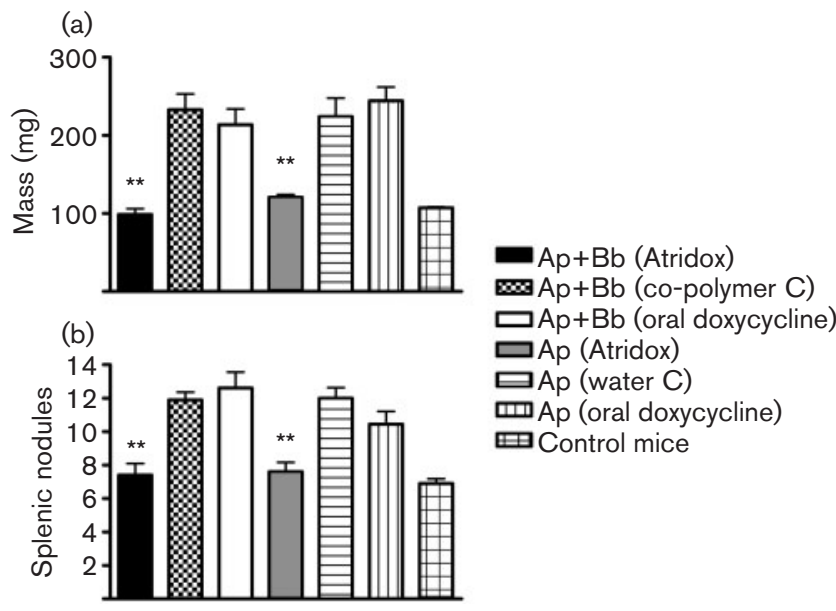

Fig. 3. (a) Comparative spleen masses of mice following tick infestation. The bars represent the mean mass per treatment group \pm SD $(n=10)$. **, $P<0.05$ between the Atridox-treated group and the co-polymer- or water-treated vehicle control mice. (b) Quantitative histopathology of spleens 21 days after tick infestation. The bars represent the mean number of lymphoid nodules per treatment group $(n=10) .{ }^{* *}, P<0.05$ between the Atridox-treated groups and the co-polymer- or water-treated vehicle control mice. Ap, A. phagocytophilum; Bb, B. burgdorferi. 
mouse model (Massung et al., 2005). Our previous studies indicated that oral delivery of $2 \mathrm{mg}$ doxycycline hyclate to mice gave peak levels of $2.43 \mu \mathrm{g}$ doxycycline (ml plasma) ${ }^{-1}$ at $8 \mathrm{~h}$ after delivery, slightly higher than the peak levels in mice given $4.2 \mathrm{mg}$ sustained-release doxycycline [1.89 $\mu \mathrm{g}$ doxycycline $(\mathrm{ml} \mathrm{plasma})^{-1}$ ] (Zeidner et al., 2004a). The present study was undertaken to determine whether a single injection of sustained-release doxycycline hyclate would be effective at preventing both $B$. burgdorferi and A. phagocytophilum delivered simultaneously by $I$. scapularis ticks. Although several in vitro studies have indicated that A. phagocytophilum is highly sensitive to doxycycline (Horowitz et al., 2001; Maurin et al., 2003), our study is the first to demonstrate that a sustained-release formulation of doxycycline is completely effective against simultaneous delivery of $B$. burgdorferi and A. phagocytophilum by questing ticks in vivo. In these studies, a single dose of oral doxycycline prevented $B$. burgdorferi infection in only $20 \%$ of the animals tested (Table 1), which was not significantly different from animals given a copolymer or a water vehicle treatment control. These results are in contrast to the $43 \%$ protection achieved in earlier studies against B. burgdorferi (Zeidner et al., 2004a), possibly due to the increased number of ticks placed on each animal $(n=10)$ to achieve dual infection. Although not analysed in this study, tick saliva has been shown previously to enhance both viral and bacterial transmission in vivo (Nuttall \& Labuda, 2004; Zeidner et al., 2002), and this effect may be limiting the effectiveness of this dose of oral doxycycline. Moreover, we do not know from these studies whether the simultaneous delivery of both $B$. burgdorferi and $A$. phagocytophilum had any effect on the subsequent metabolism of doxycycline hyclate administered orally.

Quantitative real-time PCR data (Fig. 1) indicated that, in productive A. phagocytophilum infection (both PCR- and culture-positive spleen), the copy number for $A$. phagocytophilum ranged from 800 to 10000 copies within the spleen (Fig. 1b). Moreover, these studies indicated that a threshold value of 100 copies of A. phagocytophilum in the spleen may be associated with productive infection in vivo. In animals that were below this threshold copy number (Fig. 1b), DNA could not be detected in the peripheral blood, and spleens were subsequently culture-negative when co-cultured on HL-60 cells. Likewise, no splenomegaly, lymphoid hyperplasia or splenitis (Fig. 2) was noted in those animals that were successfully treated and resisted tick-transmitted infection. In these studies, splenomegaly and lymphoid hyperplasia correlated with treatment failure (Fig. 3). The gross mass of the spleens of animals receiving either vehicle control or oral doxycycline were twice that of Atridoxtreated mice (213-244 mg versus 99-121 mg), which had spleens similar to normal, uninfected $\mathrm{C} 3 \mathrm{H} / \mathrm{HeJ}$ mice. This change in size was due to marked and expansive lymphoid hyperplasia of the white pulp with an influx of inflammatory cells, correlating with our ability to culture A. phagocytophilum on HL-60 cells.

It has been known for some time that the primary tick and vertebrate reservoirs for Lyme disease in the north-east and
Midwest regions of the USA are I. scapularis ticks and the white-footed mouse, P. leucopus (Bakken \& Dumler, 2000; McQuiston et al., 1999; Stafford et al., 1999). Other pathogens, including Babesia microti and A. phagocytophilum, have been identified in these natural reservoirs, and cotransmission of these agents with $B$. burgdorferi has been well documented (Adelson et al., 2004; Stafford et al., 1999). It has also been reported that the duration of symptoms reported by patients co-infected with these agents exceeds that for patients with B. burgdorferi infection alone, making early diagnosis and successful treatment of co-infections with appropriate antimicrobial therapy imperative (Duffy et al., 1997; Krause et al., 2002). Our studies indicate that a sustained-release formulation of doxycycline can prophylactically block co-transmission of $B$. burgdorferi and $A$. phagocytophilum by I. scapularis ticks, as indicated by PCR, culture isolation and histopathology. It remains to be tested whether similar treatment with a single injection of Atridox would be more efficacious in the elimination of an early, established co-infection than the current oral dosing schedule of doxycycline in humans. Moreover, preliminary work in mice suggests that a strategy could be devised to potentially eliminate co-infection of $B$. burgdorferi and $A$. phagocytophilum in rodent and tick reservoirs in nature with other novel formulations and delivery vehicles of doxycycline hyclate. Our laboratory is currently evaluating such strategies for use in field trials to prevent the enzootic transmission of these pathogens.

\section{REFERENCES}

Adelson, M. E., Rao, R.-V. S., Tilton, R. C., Cabets, K., Eskow, E., Fein, L., Occi, J. L. \& Mordechai, E. (2004). Prevalence of Borrelia burgdorferi, Bartonella spp., Babesia microti, and Anaplasma phagocytophila in Ixodes scapularis ticks collected in northern New Jersey. J Clin Microbiol 42, 2799-2801.

Aguero-Rosenfeld, M. E., Donnarumma, L., Zentmaier, L., Jacob, J., Frey, M., Noto, R., Carbonaro, C. A. \& Wormser, G. P. (2002). Seroprevalence of antibodies that react with Anaplasma phagocytophila, the agent of human granulocytic ehrlichiosis, in different populations in Westchester County, New York. J Clin Microbiol 40, 2612-2625.

Bakken, J. S. \& Dumler, J. S. (2000). Human granulocytic ehrlichiosis. Clin Infect Dis 31, 554-560.

CDC (2004). Lyme Disease - United States, 2001-2002. MMWR Morb Mortal Wkly Rep 53, 365-369.

Duffy, J., Pittlekow, M. R., Kolbert, C. P., Rutledge, B. J. \& Persing, D. H. (1997). Coinfection with Borrelia burgdorferi and the agent of human granulocytic ehrlichiosis. Lancet 349, 399.

Horowitz, H. W., Hsieh, T. C., Aguero-Rosenfeld, M. E., Kalantarpour, F., Chowdhury, I., Wormser, G. P. \& Wu, J. M. (2001). Antimicrobial susceptibility of Ehrlichia phagocytophila. Antimicrob Agents Chemother 45, 786-788.

Johnson, R. C., Kodner, C. B., Jurkovich, P. J. \& Collins, J. J. (1990). Comparative in vitro and in vivo susceptibilities of the Lyme disease spirochete Borrelia burgdorferi to cefuroxime and other antimicrobial agents. Antimicrob Agents Chemother 34, 2133-2136.

Krause, P. J., McKay, K., Thompson, C. A., Sikand, V. K., Lentz, R., Lepore, T., Closter, L., Christianson, D., Telford, S. R. \& other 
authors (2002). Disease-specific diagnosis of coinfecting tickborne zoonoses: babesiosis, human granulocytic ehrlichiosis, and Lyme disease. Clin Infect Dis 34, 1184-1191.

Massung, R. F., Priestley, R. A. \& Levin, M. L. (2004). Transmission route efficacy and kinetics of Anaplasma phagocytophilum infection in the white-footed mouse, Peromyscus leucopus. Vector Borne Zoonotic Dis 4, 310-318.

Massung, R. F., Zeidner, N. S., Dolan, M. C., Roellig, D., Gatitzsch, E., Troughton, D. R. \& Levin, M. L. (2005). Prophylactic use of sustainedrelease doxycycline blocks tick-transmitted infection by Anaplasma phagocytophilum in a murine model. Ann N Y Acad Sci 1063, 436-438.

Matschke, C., Isele, U., Hoogevet, P. V. \& Fahr, A. (2002). Sustainedrelease injectables formed in situ and their potential use for veterinary products. J Control Release 85, 1-15.

Maurin, M., Bakken, J. S. \& Dumler, J. S. (2003). Antibiotic susceptibilities of Anaplasma (Ehrlichia) phagocytophilum strains from various geographic areas in the United States. Antimicrob Agents Chemother 47, 413-415.

McQuiston, J. H., Paddock, C. D., Holman, R. C. \& Childs, J. E. (1999). The human ehrlichioses in the United States. Emerg Infect Dis 5, 635-642.

Nadelman, R. B., Horowitz, H. W., Hsieh, T. C., Wu, J. M., AgueroRosenfeld, M. E., Schwartz, I., Nowakowski, J., Varde, S. \& Wormser, G. P. (1997). Simultaneous human granulocytic ehrlichiosis and Lyme borreliosis. N Engl J Med 337, 27-30.

Nadelman, R. B., Nowakowski, J., Fish, D., Falco, R. C., Freeman, K., McKenna, D., Welch, P., Marcus, R., Aguero-Rosenfeld, M. E. \& other authors (2001). Prophylaxis with single-dose doxycycline for the prevention of Lyme disease after an Ixodes scapularis tick bite. N Engl J Med 345, 79-84.

Nuttall, P. A. \& Labuda, M. (2004). Tick-host interactions: salivaactivated transmission. Parasitology 129, S177-S189.

Pancholi, P., Kolbert, C. P., Mitchell, P. D., Reed, K. D., Dumler, J. S., Bakken, J. S., Telford, S. R., III \& Persing, D. H. (1995). Ixodes dammini as a potential vector of human granulocytic ehrlichiosis. J Infect Dis 172, 1007-1012.

Piesman, J. (1993). Standard system for infecting ticks (Acari: Ixodidae) with the Lyme disease spirochete Borrelia burgdorferi. J Med Entomol 30, 199-203.

Schwartz, I., Wormser, G. P., Schwartz, J. J., Cooper, D., Weissensee, P., Gazumyan, A., Zimmermann, E., Goldberg, N. S., Bittker, S. \& other authors (1992). Diagnosis of early Lyme disease by polymerase chain reaction amplification and culture of skin biopsies from erythema migrans lesions. J Clin Microbiol 30, 3082-3088.

Sinsky, R. J. \& Piesman, J. (1989). Ear punch biopsy method for detection and isolation of Borrelia burgdorferi from rodents. J Clin Microbiol 27, 1723-1727.

Stafford, K. C., Massung, R. F., Magnarelli, L. A., Ijdo, J. W. \& Anderson, J. F. (1999). Infection with agents of human granulocytic ehrlichiosis, Lyme disease, and babesiosis in wild white-footed mice in Connecticut. J Clin Microbiol 37, 2887-2892.

Wormser, G. P., Ramanathan, R., Nowakowsk, J., McKenna, D., Holmgren, D., Visintainer, P., Dornbush, R., Singh, B. \& Nadelman, R. B. (2003). Duration of antibiotic therapy for early Lyme disease. Ann Intern Med 138, 697-704.

Zeidner, N. S., Schneider, B. S., Nuncio, M. S., Gern, L. \& Piesman, J. (2002). Coinoculation of Borrelia spp. with tick salivary gland lysate enhances spirochete load in mice and is tick species-specific. J Parasitol 88, 1276-1278.

Zeidner, N. S., Brandt, K. S., Dadey, E., Dolan, M. C., Happ, C. \& Piesman, J. (2004a). Sustained-release formulation of doxycycline hyclate for prophylaxis of tick bite infection in a murine model of Lyme borreliosis. Antimicrob Agents Chemother 48, 2697-2699.

Zeidner, N. S., Carter, L. G., Monteneiri, J. A., Petersen, J. M., Schriefer, M., Gage, K. L., Hall, G. \& Chu, M. C. (2004b). An outbreak of Francisella tularensis in captive prairie dogs: an immunohistochemical analysis. J Vet Diagn Invest 16, 150-152. 